\title{
Triazatriangulene platform for self-assembled monolayers of free-standing diarylethene
}

\author{
Qing Wang ${ }^{1}$, Liang $\mathrm{Ma}^{2}$, Zhonghua Liu ${ }^{3}, \mathrm{Xu}$ Zhang ${ }^{1}$, Zhaoyang Zhang ${ }^{1}$, Zhichun Shangguan ${ }^{1}$, \\ Xianhui Huang ${ }^{1}$, Yuqing Liu ${ }^{4}$, Jingtao $\mathrm{Lv}^{2^{*}}$, Haiming Zhang ${ }^{3}$, Lifeng Chi ${ }^{3}$ and Tao $\mathrm{Li}^{{ }^{*}}$
}

Incorporating functional molecules into molecular junctions as active components is a topic of major interest in the field of molecular electronics [1-7]. Many efforts have been devoted to fabricating switchable molecular electronic devices that are responsive to external stimuli $[8,9]$. Among various stimuli (e.g., redox process [10], $\mathrm{pH}$ [11], magnetic field [12]), light offers a clean, non-invasive, low-cost and easily addressable way for triggering molecular switches in electrical devices [13,14]. Diarylethene (DAE) is considered as one of the most promising group of photo-responsive molecules due to outstanding fatigue-resistance $[15,16]$, compared with other photoswitches such as azobenzene [17] and spiropyrane [18]. Upon exposure to alternating UV and visible light, DAE isomerizes reversibly between the ring-open and closed states with distinguishing electronic properties as a result of the change in conjugated structure [19-21]. Incorporating DAE into functional molecular electronic devices possesses many advantages. First, the conformation isomerization of DAE is controlled by light irradiation with distinct wavelengths, which is promising for optical remote control devices. Second, the excellent fatigue resistance and high photochemical quantum yield of DAEs endow their devices with long lifetime and high sensitivity. Third, the structural difference between two isomers is negligible compared with other photoswitches (e.g., azobenzene and dihydroazulene), which is in favor of maintaining the morphology of the molecular junctions during photoswitching process. Benefitting from these advantages, a single-molecule DAE electrical switch with high on/off ratio, unprecedented stability, and reproducibility has been realized [22].

Self-assembled monolayers (SAMs) have been widely studied for fabricating large-area molecular junctions $[23,24]$, which provide more reproducible and robust devices than single-molecule junctions [25,26]. Thiols have been the most widely used binding groups which result in the formation of densely packed monolayers [27]. A variety of functional molecules have been immobilized on Au surface in this manner, including dihydroazulene [14], azobenzene [28], and spiropyrane [29]. However, their switching behaviors in dense SAMs are often suppressed due to the strong coupling effect between the neighboring molecules during the conformational isomerization [30]. For example, Kronemeijer et al. fabricated a photoswitching device with DAE SAMs [31], while the photo-response time $(\sim 4 \mathrm{~min})$ of the molecules in junction is apparently longer than that in solution, which is very probably due to the interference from neighboring molecules in densely packed thiolbased monolayers.

To address this issue, we utilize a non-traditional triazatriangulene (TATA) platform to assemble free-standing functional unit on Au surface. It has been reported that the TATA platforms could provide sufficient space for various functional molecules to be free-standing in the SAMs, such as azobenzene derivatives [32-34] and porphyrins [35]. In these systems, the functional units keep independent from neighbors due to large distance between them, which is a critical feature to enable the fast response of functional moieties within the monolayers [33].

In this study, we designed a photochromic molecule DAE-TATA bearing DAE as functional unit covalently linked to TATA platform, to avoid the strong coupling between neighboring DAE molecules in the SAMs (Fig.

\footnotetext{
${ }^{1}$ Department of Chemistry, Shanghai Jiao Tong University, Shanghai 200240, China

${ }^{2}$ School of Physics and Wuhan National High Magnetic Field Center, Huazhong University of Science and Technology, Wuhan 430074, China

${ }^{3}$ Institute of Functional Nano \& Soft Materials (FUNSOM), Soochow University, Suzhou 215123, China

${ }^{4}$ Institute of Semiconductors, Chinese Academy of Sciences, Beijing 100083, China

*Corresponding authors (emails: litao1983@sjtu.edu.cn (Li T); jtlu@hust.edu.cn (Lv H))
} 


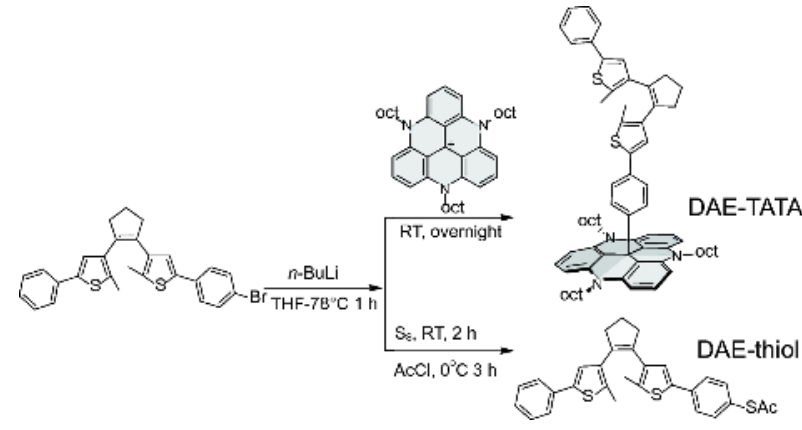

Scheme 1 Synthesis of DAE-TATA and DAE-thiol.

2a). The DAE vertically stands in the middle of a TATA platform that can be strongly adsorbed on Au surface by dispersion interaction [35].

The synthetic routes of DAE-TATA and the control molecule DAE-thiol are shown in Scheme 1. The bromoDAE was prepared as the precursor for further reactions. The corresponding lithium anions reacted with the $\mathrm{TATA}^{+}$cation and sulphur to yield DAE-TATA and DAE-thiol, respectively (see Supplementary information for more details); the latter was further protected by the acetyl.

To verify the potential of DAE-TATA being an active component in photoswitching devices, we performed DFT calculations (Fig. 1). From break-junction experiments [36], scanning tunneling microscopy (STM) measurements [37] and data from solid-state devices [31], it is clear that the tunneling current through the DAE molecular junctions is higher in the closed state than the open state. The increased conductance is ascribed to the lowering of energy gap between the highest occupied molecular orbital (HOMO) and the lowest unoccupied molecular orbital (LUMO) when the $\pi$-conjugation is extended [19]. According to calculations, the energy gap is narrowed from 3.9 to $2.5 \mathrm{eV}$ for DAE-TATA, which is sufficient for conductance switch in the corresponding electronic devices. Furthermore, the energy gaps for DAE-TATA are similar to that of DAE-thiol at both states, which indicates that thiol can be replaced by TATA while maintaining the intrinsic electronic properties for opto-electronic applications.

Upon the irradiation of UV light, the DAE-TATA solution changed from colorless to violet that restored colorless by subsequent visible light exposure, in agreement with the photoisomerization of DAE compounds, as shown in Fig. 2a. The photoswitching properties of DAETATA were studied in solution by UV-vis spectroscopy (Fig. 2b). The absorption for as prepared DAE-TATA (in the open state) was featureless but it showed a broad
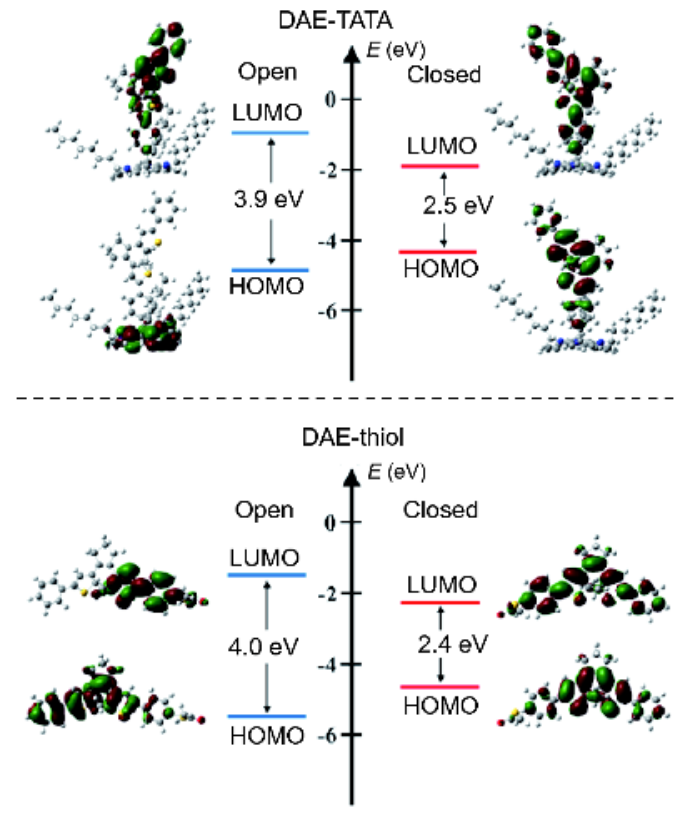

Figure 1 Energy level diagrams of DAE-TATA and DAE-thiol obtained from DFT calculations.

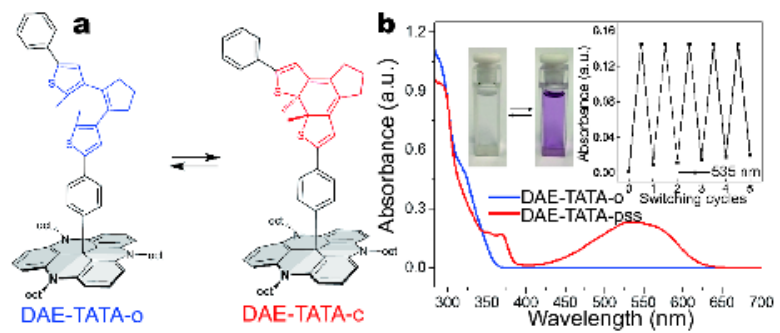

Figure 2 (a) Structural transformation of DAE-TATA. (b) UV-Vis absorption spectroscopy of DAE-TATA in toluene $\left(1 \times 10^{-5} \mathrm{~mol} \mathrm{~L}^{-1}\right)$ upon alternating irradiation with UV $(320 \mathrm{~nm})$ and visible light $(532 \mathrm{~nm})$. Inset, absorption switching cycles $(535 \mathrm{~nm})$ of DAE-TATA solution upon alternating irradiation with UV and visible light.

absorption peak at around $535 \mathrm{~nm}$ with the UV illumination, which confirmed the conversion from the ringopen isomer to closed form and finally reached the photostationary state (PSS). Meantime, the formation of closed isomer was confirmed by ${ }^{1} \mathrm{H}$ NMR and the percentage of conversion at PSS is about $40 \%$ according to the characteristic peak area ratio (Fig. S1). When visible light was applied, a complete reversal of these spectral changes was observed. DAE-thiol showed similar spectrum changes upon photoisomerization (Fig. S2). In accordance with the previous report [38], the photocyclization quantum yield of ring-open isomer $\left(\phi_{\mathrm{O}-\mathrm{C}}=0.26\right)$ is higher than the photocycloreversion quantum yield of ring-closed isomer $\left(\phi_{\mathrm{C}-\mathrm{O}}=0.009\right)$. Fig. $2 \mathrm{~b}$ inset shows the absorbance of DAE-TATA at $535 \mathrm{~nm}$ 

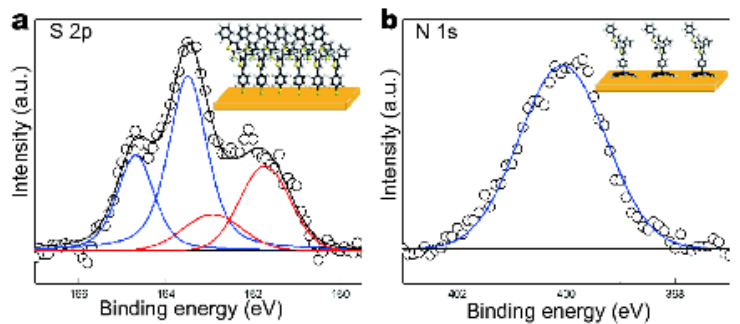

Figure 3 High-resolution XPS for (a) S 2p of DAE-thiol SAM and (b) $\mathrm{N}$ 1s of DAE-TATA SAM.

during alternating irradiation with UV and visible light. The reversible photoswitching between open and closed state was repeated for at least 5 times without obvious degradation, indicating the good fatigue resistance of DAE-TATA. Furthermore, to verify the thermal stability of the closed form, the UV-vis spectroscopy of closedstate DAE-TATA was measured before and after heating at $50^{\circ} \mathrm{C}$ for $1 \mathrm{~h}$. There is no obvious ring-opening signature in the spectrum, which confirmed that the thermal stimuli would not trigger the photo-switching behavior (Fig. S3).

After investigating the photoswitching performance of $\mathrm{DAE}$ in solution, we assembled them into SAMs on $\mathrm{Au}$ surface, which is a crucial step to build functional molecular devices. To confirm the formation and morphology of monolayers, X-ray photoelectron spectroscopy (XPS), cyclic voltammetry (CV) and STM were carried out. The SAMs were formed on clean and flat Au substrate for XPS and CV measurements. Fig. 3a depicts the $S 2 p$ spectrum for DAE-thiol SAM. The $S 2 p$ spectrum of DAE-thiol SAM could be fitted with two doublets including $S 2 p_{3 / 2}$ at $161.8 \mathrm{eV}$ attributed to the S-Au bond and $163.4 \mathrm{eV}$ assigned to the thiophene sulfur atom. These results indicated the successful attachment of DAE-thiol on $\mathrm{Au}$ substrate through S-Au bond. In Fig. 3b, the $\mathrm{N}$ 1s spectrum of DAE-TATA SAM shows a single component at $400 \mathrm{eV}$ assigned to the $\mathrm{N}$ atoms in TATA platform, which confirmed the presence of the TATA based molecules. Moreover, the $S 2 p$ spectrum proves the existence of thiophene sulfur in the DAE-TATA molecule (Fig. S4). The thickness of SAMs for DAE-TATA and DAE-thiol were estimated from the XPS measurements by $\mathrm{Au}$ attenuation (Table 1), and the values were in agreement with the theoretically calculated molecular lengths. For both thiol- and TATA-adlayer, the thickness show little difference between the open and closed state, which is consistent with the shape-constant isomerization of DAE molecules and is in favor of maintaining the morphology of molecular junctions and thus the performance of
Table 1 Thickness of SAMs: results from theoretical calculations and XPS measurements

\begin{tabular}{ccc}
\hline \multicolumn{3}{c}{ Thickness of the SAMs (nm) } \\
\hline Molecule & $\begin{array}{c}\text { Theoretically calcu- } \\
\text { lated length }\end{array}$ & XPS by Au attenuation \\
\hline DAE-thiol-o & 2.02 & 2.37 \\
DAE-thiol-c & 1.95 & 2.37 \\
DAE-TATA-o & 1.99 & 1.89 \\
DAE-TATA-c & 1.96 & 1.86 \\
\hline
\end{tabular}

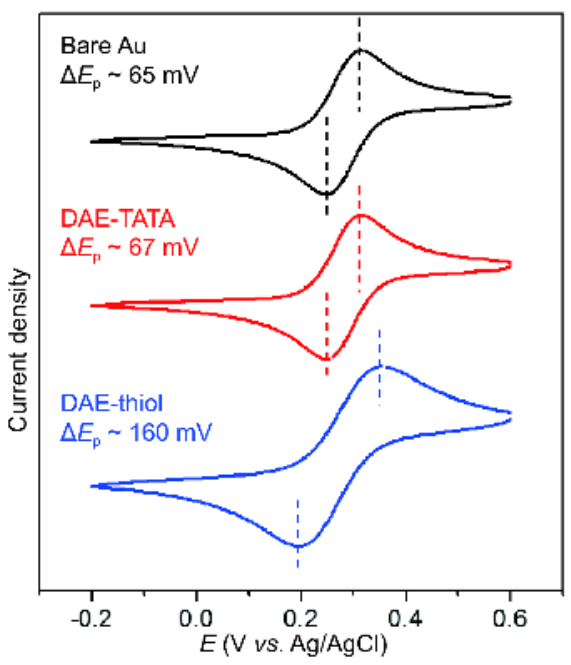

Figure $4 \mathrm{CV}$ curves of aqueous $\mathrm{K}_{3}\left[\mathrm{Fe}(\mathrm{CN})_{6}\right] / \mathrm{K}_{4}\left[\mathrm{Fe}(\mathrm{CN})_{6}\right]$ solution recorded with Au working electrodes covered by different types of SAMs (scan rate: $50 \mathrm{mV} \mathrm{s}^{-1}$ ).

molecular devices during photoswitching process.

The density and quality of the SAMs were further characterized by $\mathrm{CV}$ in $\mathrm{K}_{3} \mathrm{Fe}(\mathrm{CN})_{6}$ solution with $\mathrm{Au}$ substrates covered by SAMs as the working electrodes (Fig. 4). The $\mathrm{Fe}^{2+} / \mathrm{Fe}^{3+}$ redox signal for DAE-thiol/Au (blue) shows apparently wider peak separation $(167 \mathrm{mV})$ than that for bare Au (black), because the high-density DAE-thiol SAM blocked the access of $\mathrm{Fe}^{2+} / \mathrm{Fe}^{3+}$ species to the Au surface. On the other hand, DAE-TATA/Au (red) showed a redox peak separation that was only slightly different from bare $\mathrm{Au}$. This indicates the dilute nature of the TATA SAMs, in contrast with the thiol-SAMs.

To further illuminate the arrangement of DAE molecules within the SAMs, STM measurements were performed under ambient condition. The Au (111) single crystal was immersed in $0.1 \mathrm{mmol} \mathrm{L}^{-1}$ toluene solution of DAE-TATA for $8 \mathrm{~min}$ to prepare the SAMs. Fig. 5a depicts a highly ordered hexagonal packing of DAE-TATA monolayer. The DAE-TATA molecules show a triangular 


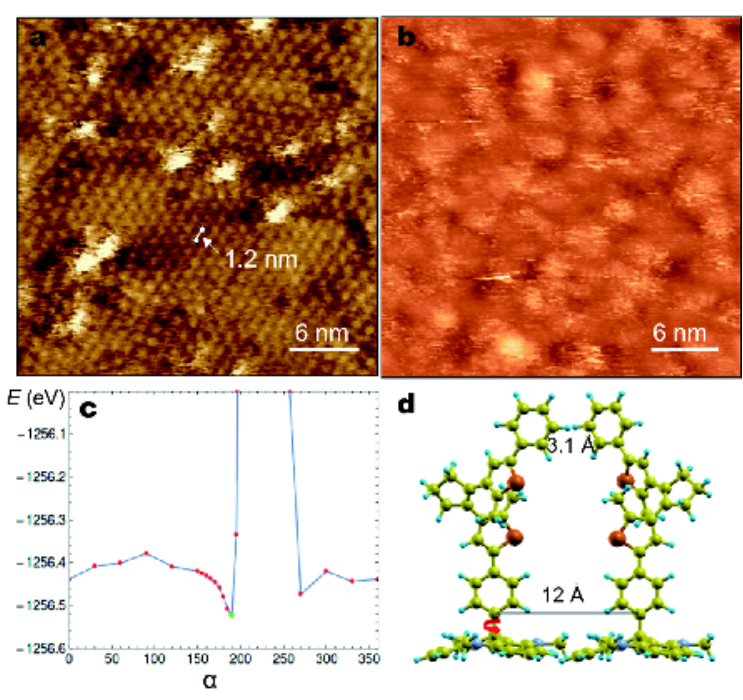

Figure 5 (a) STM image of DAE-TATA adlayer on Au (111) $\left(I_{\mathrm{t}}=8 \mathrm{pA}\right.$, $\left.V_{\text {Bias }}=0.6 \mathrm{~V}\right)$. b) STM image of DAE-thiol adlayer on Au (111) $\left(I_{\mathrm{t}}=15\right.$ pA, $V_{\text {Bias }}=0.5 \mathrm{~V}$ ). (c) Energy (E) for the rigid rotational DAE-TATA-o as a function of angle $\alpha$. The initial position that two molecules are mutually parallel was defined as $\alpha=0^{\circ}$. The local minimum in the energy curve is marked with green dot. The structure corresponding to this energy minimum is shown in Fig. 5d. For the closed state, see Fig. S6.

shape with distances of $12.0 \pm 0.2 \AA$ between neighboring molecules, which accords with the previous report [35]. There are some bright protrusions attributed to the closed state of DAE-TATA molecules which have higher conductance than as-prepared open state [37]. The adlayer pattern reveals that horizontal TATA platforms should be parallelly adsorbed on Au surface. Moreover, the photoswitchable DAE is mounted perpendicularly to the TATA platform because of the $\mathrm{sp}^{3}$ hybridization of central carbon [39]. The DAE was loosely packed with obviously enlarged distance of $12.0 \pm 0.2 \AA$. Based on the experimental data, DFT calculations were performed to estimate intermolecular interaction. It showed that at the energy minimum during the azimuthal rotation of DAETATA, the nearest distance between the laterally extending $\mathrm{H}$ atoms was $3.1 \AA$ (Fig. $5 \mathrm{c}$, d). At this distance, the steric hindrance was prevented, suggesting little intermolecular interaction in the SAM, which may preferably contribute to a fast photo-response of molecules.

For comparison, the DAE-thiol SAM was prepared by immersing $\mathrm{Au}$ (111) in $0.1 \mathrm{mmol} \mathrm{L}^{-1} \mathrm{THF}$ solution for 2 days. In contrast with well-ordered packing of DAETATA, no ordered structures and individual molecules can be observed from the STM image of the DAE-thiol SAM (Fig. 5b). Typical distance between neighboring thiol-molecules in SAMs (e.g., dioctadecyl sulfide [40], terphenylthiolate [41] and azobenzene-thiol [42]) was reported to be around $5 \AA$. Therefore the recorded indistinct image was probably due to the overlap of electron cloud between neighboring molecules in monolayer as a result of the large lateral size of DAE group and a densely packed arrangement of thiol-based SAM.

In addition to the ordered and dilute arrangement, TATA-based SAM can be more conveniently prepared (8 min) and is more stable in air than the thiol-system (requiring 2 days for the self-assembly process with thiols easy to be oxidized [43]). These features are very attractive for high-yield fabrication of functional molecular electronic devices. On this basis, solid-state SAM devices were fabricated to detect the photo-response characteristics of the molecules in micropore junctions (Fig. S5a). $90 \%$ of the measured junctions (more than 20 junctions) showed obvious conductance increase (up to 25\%) upon UV irradiation (Fig. S5b), indicating the molecular signature of DAE switches. More detailed investigations on SAM devices are underway.

In summary, we designed and synthesized a novel photochromic molecule, DAE-TATA with DAE-thiol as a control system. The photoswitching behavior of DAETATA was confirmed by solution UV-vis and the selfassembled monolayers on Au surface were studied using XPS, CV and STM. The results showed that DAE-TATA assembled on gold surface provided sufficient spatial freedom for individual DAE without the interference from neighboring molecules, which was also supported by DFT calculations. We believe this study provides a desirable molecular candidate for promising applications in functional molecular electronic devices.

Received 31 January 2018; accepted 2 April 2018; published online 18 April 2018

1 Li T, Hu W, Zhu D. Nanogap electrodes. Adv Mater, 2010, 22 : 286-300

2 Xiang D, Wang X, Jia C, et al. Molecular-scale electronics: from concept to function. Chem Rev, 2016, 116: 4318-4440

3 Feng L, Dong $\mathrm{H}$, Li Q, et al. Tuning crystal polymorphs of a $\pi$ extended tetrathiafulvalene-based cruciform molecule towards high-performance organic field-effect transistors. Sci China Mater, 2016, 60: 75-82

4 Zhang ZY, Li T. Single-chain and monolayered conjugated polymers for molecular electronics. Chin Chem Lett, 2016, 27: 12091222

5 Sun L, Diaz-Fernandez YA, Gschneidtner TA, et al. Single-molecule electronics: from chemical design to functional devices. Chem Soc Rev, 2014, 43: 7378-7411

6 Zhang X, Li T. Molecular-scale electronics: From device fabrication to functionality. Chin Chem Lett, 2017, 28: 2058-2064 
7 Lv X, Wang H, Meng L, et al. Highly efficient inverted organic light-emitting diodes based on thermally activated delayed fluorescence. Sci China Mater, 2016, 59: 421-426

8 Gui B, Meng X, Chen Y, et al. Reversible tuning hydroquinone/ quinone reaction in metal-organic framework: immobilized molecular switches in solid state. Chem Mater, 2015, 27: 6426-6431

9 Gui B, Meng Y, Xie Y, et al. Immobilizing organic-based molecular switches into metal-organic frameworks: a promising strategy for switching in solid state. Macromol Rapid Commun, 2018, 39: 1700388-1700399

10 Wang Z, Dong H, Li T, et al. Role of redox centre in charge transport investigated by novel self-assembled conjugated polymer molecular junctions. Nat Commun, 2015, 6: 7478

11 Guo X, Small JP, Klare JE, et al. Covalently bridging gaps in singlewalled carbon nanotubes with conducting molecules. Science, 2006, 311: 356-359

12 Sanvito S. Molecular spintronics. Chem Soc Rev, 2011, 40: 33363355

13 Fihey A, Perrier A, Browne WR, et al. Multiphotochromic molecular systems. Chem Soc Rev, 2015, 44: 3719-3759

14 Li T, Jevric M, Hauptmann JR, et al. Ultrathin reduced graphene oxide films as transparent top-contacts for light switchable solidstate molecular junctions. Adv Mater, 2013, 25: 4164-4170

15 Irie M. Diarylethenes for memories and switches. Chem Rev, 2000, 100: $1685-1716$

16 Liu SJ, Yang CJ, Xu WJ, et al. Effect of metal centres and substituents on the structure and optoelectronic properties of diarylethene compounds: A theoretical study. Sci China Chem, 2012, 56: 137-147

17 Yuan R, Ren $\mathrm{H}, \mathrm{He} \mathrm{H}$, et al. Targeted synthesis of porous aromatic frameworks with stimuli-responsive adsorption properties. Sci China Mater, 2015, 58: 38-43

18 Jang AR, Jeon EK, Kang D, et al. Reversibly light-modulated dirac point of graphene functionalized with spiropyran. ACS Nano, 2012, 6: 9207-9213

19 Jia C, Wang J, Yao C, et al. Conductance switching and mechanisms in single-molecule junctions. Angew Chem Int Ed, 2013, 52: 8666-8670

20 Irie M, Fukaminato T, Matsuda K, et al. Photochromism of diarylethene molecules and crystals: memories, switches, and actuators. Chem Rev, 2014, 114: 12174-12277

21 Zhang J, Tian H. The endeavor of diarylethenes: new structures, high performance, and bright future. Adv Opt Mater, 2018, 42: 1701278

22 Jia C, Migliore A, Xin N, et al. Covalently bonded single-molecule junctions with stable and reversible photoswitched conductivity. Science, 2016, 352: 1443-1445

23 Casalini S, Bortolotti CA, Leonardi F, et al. Self-assembled monolayers in organic electronics. Chem Soc Rev, 2017, 46: 40-71

24 Jeong H, Kim D, Xiang D, et al. High-yield functional molecular electronic devices. ACS Nano, 2017, 11: 6511-6548

25 Akkerman HB, Blom PWM, de Leeuw DM, et al. Towards molecular electronics with large-area molecular junctions. Nature, 2006, 441: $69-72$

26 Li T, Hauptmann JR, Wei Z, et al. Solution-processed ultrathin chemically derived graphene films as soft top contacts for solidstate molecular electronic junctions. Adv Mater, 2012, 24: 13331339

27 Vericat C, Vela ME, Benitez G, et al. Self-assembled monolayers of thiols and dithiols on gold: new challenges for a well-known sys- tem. Chem Soc Rev, 2010, 39: 1805-1834

28 Mativetsky JM, Pace G, Elbing M, et al. Azobenzenes as lightcontrolled molecular electronic switches in nanoscale metal-molecule-metal junctions. J Am Chem Soc, 2008, 130: 9192-9193

29 Kumar S, van Herpt JT, Gengler RYN, et al. Mixed monolayers of spiropyrans maximize tunneling conductance switching by photoisomerization at the molecule-electrode interface in EGaIn junctions. J Am Chem Soc, 2016, 138: 12519-12526

30 van der Molen SJ, Liao J, Kudernac T, et al. Light-controlled conductance switching of ordered metal-molecule-metal devices. Nano Lett, 2009, 9: 76-80

31 Kronemeijer AJ, Akkerman HB, Kudernac T, et al. Reversible conductance switching in molecular devices. Adv Mater, 2008, 20: $1467-1473$

32 Jacob H, Ulrich S, Jung U, et al. Monitoring the reversible photoisomerization of an azobenzene-functionalized molecular triazatriangulene platform on $\mathrm{Au}(111)$ by IRRAS. Phys Chem Chem Phys, 2014, 16: 22643-22650

33 Jung U, Kuhn S, Cornelissen U, et al. Azobenzene-containing triazatriangulenium adlayers on $\mathrm{Au}(111)$ : structural and spectroscopic characterization. Langmuir, 2011, 27: 5899-5908

34 Kuhn S, Baisch B, Jung U, et al. Self-assembly of triazatriangulenium-based functional adlayers on $\mathrm{Au}(111)$ surfaces. Phys Chem Chem Phys, 2010, 12: 4481-4487

35 Otte FL, Lemke S, Schütt $\mathrm{C}$, et al. Ordered monolayers of freestanding porphyrins on gold. J Am Chem Soc, 2014, 136: 1124811251

36 Dulić D, van der Molen SJ, Kudernac T, et al. One-way optoelectronic switching of photochromic molecules on gold. Phys Rev Lett, 2003, 91: 207402

37 Katsonis N, Kudernac T, Walko M, et al. Reversible conductance switching of single diarylethenes on a gold surface. Adv Mater, 2006, 18: 1397-1400

38 Chai X, Fu YX, James TD, et al. Photochromism and molecular logic gate operation of a water-compatible bis-glycosyl diarylethene. Chem Commun, 2017, 53: 9494-9497

39 Wei Z, Wang X, Borges A, et al. Triazatriangulene as binding group for molecular electronics. Langmuir, 2014, 30: 14868-14876

40 Noh J, Kato HS, Kawai M, et al. Surface and adsorption structures of dialkyl sulfide self-assembled monolayers on $\mathrm{Au}(111)$. J Phys Chem B, 2002, 106: 13268-13272

41 Bashir A, Azzam W, Rohwerder M, et al. Polymorphism in selfassembled terphenylthiolate monolayers on $\mathrm{Au}(111)$. Langmuir, 2013, 29: 13449-13456

42 Pace G, Ferri V, Grave C, et al. Cooperative light-induced molecular movements of highly ordered azobenzene self-assembled monolayers. Proc Natl Acad Sci USA, 2007, 104: 9937-9942

43 Hutt DA, Cooper E, Leggett GJ. Structure and mechanism of photooxidation of self-assembled monolayers of alkylthiols on silver studied by XPS and static SIMS. J Phys Chem B, 1998, 102: 174-184

Acknowledgements The authors acknowledge the financial support from the National Key Research and Development Program of China (2017YFA0207500), the National Natural Science Foundation of China (51673114) and Shanghai Science and Technology Committee (17ZR1447300).

Author contributions Li T designed the experiments; Wang Q synthesized the molecules and measured performance with support from 
Shangguan $\mathrm{Z}$ and Huang X. Lv J, Ma L and Zhang X contributed to the DFT calculations. Chi L, Zhang $\mathrm{H}$ and Liu Z contributed to the STM measurement. Liu Y fabricated and measured the SAM devices. Wang Q wrote the paper with support from Zhang $\mathrm{Z}$ and Li T. All authors contributed to the general discussion.
Conflict of interest The authors declare that they have no conflict of interest.

Supplementary information Experimental details and supporting data are available in the online version of the paper.

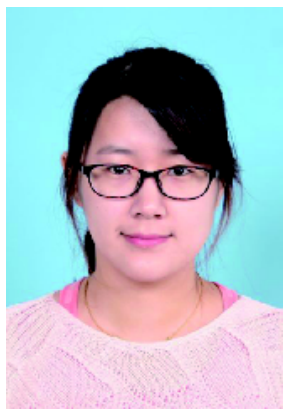

Qing Wang is a master student in the Department of Chemistry, School of Chemistry and Chemical Engineering, Shanghai Jiao Tong University, China, under the surpervision of Prof. Tao Li. Her current research interests focus on molecular electronics.

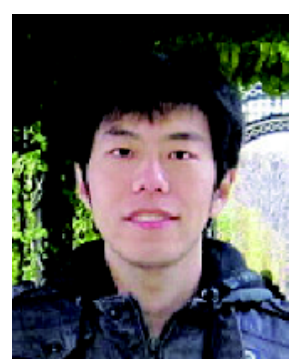

Tao Li is an Associate Professor in the School of Chemistry and Chemical Engineering, Shanghai Jiao Tong University. $\mathrm{He}$ received his $\mathrm{PhD}$ from the Institute of Chemistry, Chinese Academy of Sciences in 2010. Then he joined the University of Copenhagen as a postdoc fellow. In 2013, he was appointed as an Assistant Professor in the Department of Chemistry, University of Copenhagen. He returned to China and joined Shanghai Jiao Tong University as an Associate Professor in 2015. His research mainly focuses on molecular electronics, especially on the fabrication of solid-state molecular scale devices.

\section{基于三角大环针定基团的二芳基乙烯自组装单分子层}

王晴 ${ }^{1}$,马亮 ${ }^{2}$, 刘中华 ${ }^{3}$, 张旭 ${ }^{1}$, 张召阳 ${ }^{1}$, 上官之春 ${ }^{1}$, 黄先会 ${ }^{1}$, 刘雨晴 ${ }^{4}$, 吕京涛 ${ }^{2 *}$, 张海明 ${ }^{3}$, 迟力峰 ${ }^{3}$ 李涛 ${ }^{*}$

摘要 功能基团和针定基团的合理设计是分子器件发展的前提. 迄今为止, 颈基作为针定基团被广泛应用于电荷传输测试中. 然而, 由金硫 键所组装的分子层往往十分致密, 这通常会阻碍功能基团的活性进而影响器件性能. 本论文利用三角大环(TATA)平面作为新的针定基团, 将二芳基乙烯分子装配在金表面, 形成取向均一的、松散的单分子层. 本文通过多项测试表征了该分子及其对比体系(颈基作为针定基团) 在溶液中以及分子层中的性质. 结果表明, TATA作为针定基团可以为二芳基乙烯分子提供充足的自由体积, 有效阻隔了分子层中相邻分 子的干扰. 因此, TATA二芳基乙烯分子体系对于开发高性能的分子光开关器件具有重要意义. 